\title{
QUALITY ASSESSMENT FOR POLYGON GENERALIZATION
}

\author{
E. S. Podolskaya ${ }^{\text {a }}$, K.-H. Anders ${ }^{\text {b }}$, J.-H. Haunert ${ }^{\text {b }}$, M. Sester ${ }^{\text {b }}$ \\ ${ }^{\mathrm{a}}$ Chair of cartography, Cartographic faculty, Moscow state university of geodesy and cartography, \\ Gorohovskiy by-street 4, 105064 Moscow, Russia - podols_kate@mail.ru \\ ${ }^{\mathrm{b}}$ Institute of cartography and geoinformatics, Leibniz Universität Hannover, \\ Appelstrase 9a, 30167 Hannover, Germany - (Karl-Heinrich.Anders, Jan.Haunert, Monika.Sester)@ikg.uni-hannover.de
}

\section{Commission 2, WG 7}

KEY WORDS: generalization, polygon, data quality assessment

\begin{abstract}
:
Different methods for map generalization have been developed. However, there are only a few publications that discuss the automatic quality assessment of generalized maps. This problem becomes crucial when the usefulness of a map has to be evaluated or different methods need to be compared, e.g. to find the best algorithm for a specific application. In this paper we present a new approach for the quality assessment of generalized polygons. In particular the simplification of buildings and the generalization of polygons that represent land use in a topographic database are discussed. Our approach distinguishes between two aims of generalization: Reducing the amount of data and keeping the map similar to the input map. A measure of quality that defines a compromise between these conflicting objectives is introduced. The proposed method was tested for a German cadastral data set and the official German topographic database ATKIS.
\end{abstract}

\section{INTRODUCTION}

The quality of a map can be understood as its ability to satisfy the needs of users (TSNIIGAiK, 2003). Evaluating and ensuring the quality is one of the primary goals of map generalization (Cheng, Li, 2006). Experts suggest several directions, for example Müller et al. (1995) proposed to clarify the expectations in terms of data quality and to analyze the potential errors introduced by using digitized maps in a GIS. The problem of quality assessment in generalization is not new, it has been tackled in several approaches (e.g. van Smaalen, 2003; Galanda, 2003; Bard, 2004; Skopeliti, Tsoulos, 2001).

The aims of our paper are: (1) To propose a method for data quality assessment of polygon generalization by adapting approaches of different authors; (2) to apply the method to buildings in cadastral data sets and to areas of different land cover in a topographic database.

The topographic database contains digital landscape models (DLM) of four different scales which were taken from the German "Authoritative Topographic-Cartographic Information System" (ATKIS) (www.atkis.de). These DLM are called Basis-DLM (1:25.000), DLM50 (1:50.000), DLM250 (1:250.000), and DLM1000 (1:1.000.000). We used DLM50 and DLM250 for our investigations. Buildings at scale 1:10.000 were taken from the German cadastre. This data set was generalized to scale 1:20.000 with the software CHANGE, developed at the ikg (www.ikg.unihannover.de).

Polygons are used to represent various anthropogenous and natural objects (types of land cover, vegetation, etc.) in digital data sets.

Polygons representing areas of land cover differ from polygons representing buildings in several ways. For example, landcover polygons have many vertices and a complex, irregular shape, while buildings often have rectangular shapes.

The paper is organized according to the following structure. After the introduction we describe related work, i.e., elements of quality assessment, existing ideas on quality assessment of polygon generalization and measures for polygon generalization. The third section is devoted to two aims of generalization. Our method of integrating different objectives into a single quality measure is considered in the fourth section. The application of our method to a German cadastral data set and the official German topographic database ATKIS is presented and discussed in the next section. The paper is finished with some conclusions and directions for future research.

\section{RELATED WORK}

Data quality assessment requires three steps: Specification of requirements, definition of data quality measures and evaluation of data quality (Joao, 1998). Up to now, researchers have focused mainly on data quality requirements and data quality measures.

Elements of data quality assessment have been discussed in different papers and books, and are also standardized in national and international standards. Mayberry (2002) have proposed the following components: Accuracy, integrity, consistency, completeness, validity, timeliness, accessibility. The factors affecting the quality of spatial data are shown in (Burrough, McDonnell, 1998): Currency, completeness, consistency, accessibility, accuracy and precision, sources of errors in data, sources of errors in derived data and in the results of modeling and analysis. Guptill, Morrison (1995) described the elements of data quality: Lineage, accuracy (positional, attribute and semantic accuracy), completeness, logical consistency, temporal information. Quantitative (completeness, accuracy, 
correctness of identification of objects, logic coordination of structure, representation of objects) and qualitative (purpose, lineage or source of data) indicators are used for quality assessment in (Kolokolova, 2005). Thus, researchers suggest and use identical elements for the characteristic of data quality assessment. Also there is a division into quantitative and qualitative attributes.

The data quality concept in map generalization has been described in the following components: Object completeness of target scale to the initial scale as well as details of the qualitative characteristic of the phenomenon (Garaevskaya, Malusova, 1990).

In recent years some investigations for developing the evaluation model with quantitative parameters are undertaken. Bard and Ruas (2004) define the quality using the deviation from a given ideal. In this way, specifications for ideals are used (e.g. minimum size for legibility) and compared with the generalized situation. The ideal is defined using scale dependent functions.

A paper by Frank and Ester (2006) describes the method of quality assessment of a whole map. For a comparison of two maps they use values for a shape, location and information. The approach takes into account changes in individual objects in the form of shape similarity, groups of objects using the location similarity and changes across the entire map using semantic content similarity.

But despite of this research, we do not have a comprehensive investigation of quality assessment in polygon generalization. First of all, we should conclude that in the majority of suggested methods various levels of the data quality assessment from one separate object up to a whole map have been proposed: Macro (for the map), meso (for groups of objects) and micro (for individual objects). Such a concept is e.g. used by Peter (2001). Secondly, the evaluation of generalization quality depends on a choice of an optimal set of these measures. There is a large number of measures for polygonal maps which can be used for map quality evaluation. A very detailed description of such measures is presented in Peter (2001). We can give here only a very brief classification of these measures into seven classes with their relation to the map levels:

- Size (micro, meso, macro): Absolute and relative geometric properties of a polygon, e.g. area or perimeter.

- Shape (micro, meso, macro): For instance shape descriptors could be compactness, convexity, principal components (Peura, Iivarinen, 1997), or Fourier descriptors (Zahn, Roskies, 1977).

- Distance (micro, meso): Geometric proximity of polygons, e.g. Hausdorff distance.

- Topology (micro, meso): Occurrence of selfintersections, orientation changes, aggregation or separation.

- Density (meso, macro): Preservation of the distribution of polygons, number of polygons in a certain area, or covered area by polygons in a certain region.

- Pattern (meso, macro): Preservation of patterns, e.g. alignments, grid-, ring-, or star-structures (Anders 2006; Heinzle, Anders, Sester, 2006).

- Semantic/Information (meso, macro): Based on hierarchical ontologies or concept hierarchies it is possible to include semantic into similarity measures (Anders, 2004; Rodriguez and Egenhofer, 2004).

Obviously, there is a large variety of measures to quantify the quality of a polygon generalization. Some of these measures are difficult to assess and implement (e.g. patterns). In this paper we define some new polygon measures on micro level, but with the focus of an integrated quality measure.

\section{TWO AIMS OF GENERALIZATION}

In general, there are two conflicting aims in generalization: on the one hand, the amount of data has to be reduced; on the other hand the resulting map has to be similar to the original one. We try to use measures for these two goals and integrate them using a simple weighted addition.

\subsection{Reducing the amount of data}

The amount of map information decreases when its scale is reduced. We have considered two types of reduction: Reducing the amount of objects (polygons) and reducing the amount of detail (vertices) of individual objects.

Reducing the amount of polygons can be achieved with the following generalization operations:

a) A polygon is not represented in another scale according to rules for this scale (elimination);

b) A polygon is merged with another polygon (aggregation).

The degree of reduction due to these reasons is shown in Table 1 for land cover polygons from ATKIS DLMs. It shows that there is a considerable degree of reduction.

Table 1. Amount of land cover polygons from DLMs

\begin{tabular}{|l|c|c|c|c|}
\hline $\begin{array}{c}\text { Layer } \\
\text { of DLM }\end{array}$ & $\begin{array}{c}\text { Basis } \\
\text { DLM }\end{array}$ & $\begin{array}{c}\text { DLM } \\
50\end{array}$ & $\begin{array}{c}\text { DLM } \\
250\end{array}$ & $\begin{array}{c}\text { DLM } \\
1000\end{array}$ \\
\hline Farmland & 544 & 457 & 12 & 5 \\
\hline Grassland & 977 & 739 & - & - \\
\hline Garden & 727 & 429 & 12 & - \\
\hline Heathland & 164 & 2 & - & - \\
\hline Moor & 33 & 4 & - & - \\
\hline Total number & 2455 & 1631 & 24 & 5 \\
\hline
\end{tabular}

Reducing the number of polygon vertices is mainly achieved using the simplification of the outline by either removing vertices or approximating it with approximation functions. This reduction has to take the type of object into account. For example, for buildings it means that their rectangular form has to be preserved.

As a matter of fact, map scale is the primary factor influencing both kinds of reduction. So, in small-scale maps the degree of reduction in number of objects and amount of detail of objects is much higher than in large-scale maps.

\subsection{Keeping the map similar to the input map}

Keeping the map similar to the input map is the second main goal of map generalization. Similarity can be defined in 
terms of object size before and after generalization, or the respective perimeter values.

Another measure for the analysis of shape similarity based on the stepping turning function is described by Frank and Ester (2006). This function describes a shape by its perimeter vs. slope. The $\mathrm{x}$-coordinate in this function denotes the distance along the perimeter; the y-coordinate denotes the value of the slope. The similarity value can then be computed using the two turning functions of the polygon before and after generalization:

$V_{T F}=1-\frac{\operatorname{Area}\left(T F_{1} \Delta T F_{2}\right)}{\operatorname{Max}\left[\operatorname{Area}\left(T F_{1}\right), \operatorname{Area}\left(T F_{2}\right)\right]}$

with

$T F_{1} \quad$ being the shape between the $\mathrm{x}$-axis and the turning function of the polygon in map M1,

$T F_{2} \quad$ being the shape between the $\mathrm{x}$-axis and the turning function of the polygon in map M2, and

$T F_{1} \Delta T F_{2}$ being the symmetric difference of $T F_{1}$ and $T F_{2}$, i. e., the shape between both turning functions.

Obviously, if both polygons fully overlap, then $\operatorname{Area}\left(T F_{1} \Delta T F_{2}\right)=0 \quad$ and $\quad V_{T F}=1, \quad$ otherwise $\operatorname{Area}\left(T F_{1} \Delta T F_{2}\right)>0$ and $V_{T F}<1$. In either case $V_{T F} \geq 0$, since $\operatorname{Area}\left(T F_{1} \Delta T F_{2}\right) \leq \operatorname{Max}\left[\operatorname{Area}\left(T F_{1}\right)\right.$, Area $\left.\left(T F_{2}\right)\right]$.

To visualize the measure, a test polygon with 12 vertices has been constructed. M1 means initial map, M21 is a first version of generalization, and M22 is a second version of generalization. Using formula (1) we received $V_{T F}=0.8986$ (from M1 to M21) and $V_{T F}=0.9031$ (from M1 to M22).

In our test polygons on maps M21 and M22 are very similar. Therefore, only a small difference is obtained for their similarity measures. The results of Frank and Ester (2006) showed that this measure is a good indicator for the comparison of polygons in different scales.

\section{INTEGRATION OF DIFFERENT MEASURES}

In order to combine two opposite goals of generalization, we integrate the measures described above:

1. Reduction of polygon vertices $\left(V_{N}\right)$

2. Keeping the map similar to the input map is based on:

- Area of polygon $\left(V_{A}\right)$,

- Perimeter of polygon $\left(V_{P}\right)$,

- Turning function $\left(V_{T F}\right)$.

For the quality assessment we use the values of parameters $V_{T F}, V_{N}, V_{A}$ and $V_{P}$ from Equations (1)-(4). Values equal or close to ' 1 ' indicated good quality, whereas bad quality is denoted with values equal or close to ' 0 '.

$$
\begin{aligned}
& V_{N}=\frac{\left|N_{2}-N_{1}\right|}{\operatorname{Max}\left(N_{1}, N_{2}\right)} \\
& V_{A}=1-\frac{\left|\operatorname{Area}\left(p_{2}\right)-\operatorname{Area}\left(p_{1}\right)\right|}{\operatorname{Max}\left[\operatorname{Area}\left(p_{2}\right), \operatorname{Area}\left(p_{1}\right)\right]} \\
& V_{P}=1-\frac{\left|\operatorname{Perimeter}\left(p_{2}\right)-\operatorname{Perimeter}\left(p_{1}\right)\right|}{\operatorname{Max}\left[\operatorname{Perimeter}\left(p_{2}\right), \operatorname{Perimeter}\left(p_{1}\right)\right]}
\end{aligned}
$$

with

$p_{1}$ and $p_{2}$ being two corresponding polygons having $N_{1}$ and $N_{2}$ vertices, respectively.

Normally, generalization will result in a reduction of polygon vertices, i.e., $N_{1} \geq N_{2}$.

In this case, Equation (2) simplifies to

$$
V_{N}=1-\frac{N_{2}}{N_{1}}
$$

The overall quality measure of polygons is calculated as a weighted sum of these measures:

$V=c_{T F} \cdot V_{T F}+c_{N} \cdot V_{N}+c_{A} \cdot V_{A}+c_{P} \cdot V_{P}$,

with $c_{T F}+c_{N}+c_{A}+c_{P}=1$,

where $c_{T F}, c_{N}, c_{A}$, and $c_{P}$ are the weights of the different quality measures.

There are two approaches to using the weights in the quality assessment of generalization. First, the biggest weight can be given to the parameter which is the most important for the user, and results in good quality of this parameter (Frank, Ester, 2006). This approach is called a direct task.

Secondly, we can assign arbitrary weights to all parameters. Then we receive results for different weight combinations and can make a choice as to what is the most preferable variant with respect to the visual quality of the result. This approach can be called a return task.

Table 2 shows possible sets of weights. The rational behind Variant 1 is the fact that the two opposing goals reduction (parameter $V_{N}$ ) and preservation (parameters $V_{A}, V_{P}, V_{T F}$ ) are weighted equally.

Obviously, the number of variants is not limited to the presented variants. The defined weight sets have been tested for buildings and land cover polygons. 
The International Archives of the Photogrammetry, Remote Sensing and Spatial Information Sciences, Vol. 34, Part XXX

Table 2. Variants of weights

\begin{tabular}{|l|c|c|c|c|}
\hline & $c_{N}$ & $c_{A}$ & $c_{P}$ & $c_{T F}$ \\
\hline Variant 1 & 0.5 & 0.167 & 0.167 & 0.167 \\
\hline Variant 2 & 0.167 & 0.5 & 0.167 & 0.167 \\
\hline Variant 3 & 0.167 & 0.167 & 0.5 & 0.167 \\
\hline Variant 4 & 0.167 & 0.167 & 0.167 & 0.5 \\
\hline
\end{tabular}

\section{IMPLEMENTATION OF OUR APPROACH}

\subsection{Buildings in scales 1:10.000 and 1:20.000}

The buildings from the cadastral data set (original scale approx. 1:10.000) have been generalized using the software package CHANGE. This generalization software has been developed for building generalization in large-scale (1:1.000-1:25.000) maps. It includes: (1) Preprocessing and adjustment of data, (2) generalization of contours and (3) the aggregation of neighboring buildings. There is the possibility to control which objects can be aggregated based on semantic criteria.

The measures used in this paper are all based on a comparison of properties of a polygon before and after generalization. In case of a reduction in the number of objects, i.e., elimination and aggregation, these measures can not be applied directly.

In case of elimination of an object the values for the reduction are optimal $\left(V_{N}=1\right)$, whereas the values for shape similarity are $0\left(V_{A}=V_{P}=V_{T F}=0\right)$.

For aggregated buildings we calculated $V_{N}, V_{A}$ and $V_{P}$ by defining $N_{1}$, Area $\left(p_{1}\right)$ and Perimeter $\left(p_{1}\right)$ in Equations (2)(4) to be the sums of these values for individual components in the original scale. The turning function, however, was derived using a manually aggregated object.

We have analyzed three samples of buildings in scales 1:10.000 and 1:20.000 with different structure (Figure 1).

The minimal quality $\left(V_{\min }\right)$, the maximal quality $\left(V_{\max }\right)$, their difference $(\Delta)$ and the average quality $\left(V_{a}\right)$ of each sample are presented in Table 3. Together, these parameters indicate the quality of each sample.

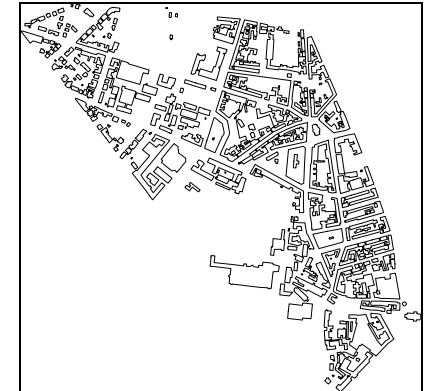

(a)

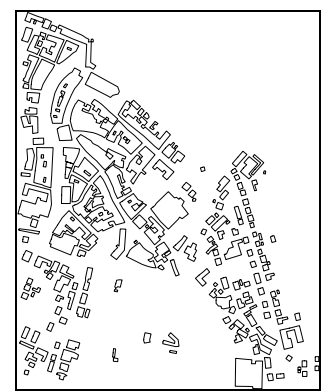

(b)

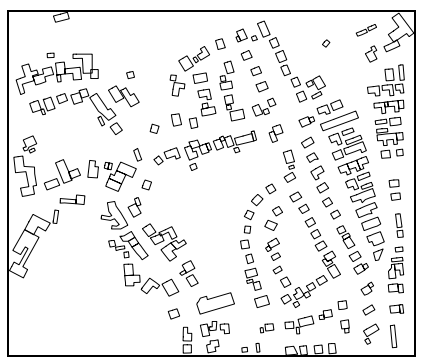

(c)

Figure 1. Source dataset from cadastre (scale 1:10.000). Samples of three types: city area, two types of rural areas

Table 3. Test results: Minimum, maximum and average quality of building samples

\begin{tabular}{|c|c|c|c|c|c|c|}
\hline & \multicolumn{2}{|c|}{ SAMPLE (A) } & \multicolumn{2}{|c|}{ SAMPLE (B) } & \multicolumn{2}{|c|}{ SAMPLE (C) } \\
\hline & $V_{\min }$ & $V_{\max }$ & $V_{\min }$ & $V_{\max }$ & $V_{\text {min }}$ & $V_{\max }$ \\
\hline \multirow{2}{*}{$\begin{array}{c}\text { Variant } \\
1\end{array}$} & 0.388 & 0.843 & 0.360 & 0.690 & 0.421 & 0.673 \\
\hline & \multicolumn{2}{|c|}{$\Delta=0.455$} & \multicolumn{2}{|c|}{$\Delta=0.330$} & \multicolumn{2}{|c|}{$\Delta=0.252$} \\
\hline$V_{a}$ & \multicolumn{2}{|c|}{0.607} & \multicolumn{2}{|c|}{0.549} & \multicolumn{2}{|l|}{0.528} \\
\hline \multirow{2}{*}{$\begin{array}{c}\text { Variant } \\
2\end{array}$} & 0.564 & 0.920 & 0.476 & 0.876 & 0.643 & 0.863 \\
\hline & \multicolumn{2}{|c|}{$\Delta=0.356$} & \multicolumn{2}{|c|}{$\Delta=0.400$} & \multicolumn{2}{|c|}{$\Delta=0.220$} \\
\hline$V_{a}$ & \multicolumn{2}{|c|}{0.828} & \multicolumn{2}{|c|}{0.799} & \multicolumn{2}{|l|}{0.810} \\
\hline \multirow{2}{*}{$\begin{array}{c}\text { Variant } \\
3\end{array}$} & 0.660 & 0.920 & 0.409 & 0.876 & 0.700 & 0.863 \\
\hline & \multicolumn{2}{|c|}{$\Delta=0.260$} & \multicolumn{2}{|c|}{$\Delta=0.467$} & \multicolumn{2}{|c|}{$\Delta=0.163$} \\
\hline$V_{a}$ & \multicolumn{2}{|c|}{0.825} & \multicolumn{2}{|c|}{0.807} & \multicolumn{2}{|l|}{0.817} \\
\hline \multirow{2}{*}{$\begin{array}{c}\text { Variant } \\
4\end{array}$} & 0.592 & 0.909 & 0.651 & 0.865 & 0.722 & 0.835 \\
\hline & \multicolumn{2}{|c|}{$\Delta=0.317$} & \multicolumn{2}{|c|}{$\Delta=0.214$} & \multicolumn{2}{|c|}{$\Delta=0.113$} \\
\hline$V_{a}$ & \multicolumn{2}{|c|}{0.834} & \multicolumn{2}{|c|}{0.817} & \multicolumn{2}{|l|}{0.814} \\
\hline
\end{tabular}




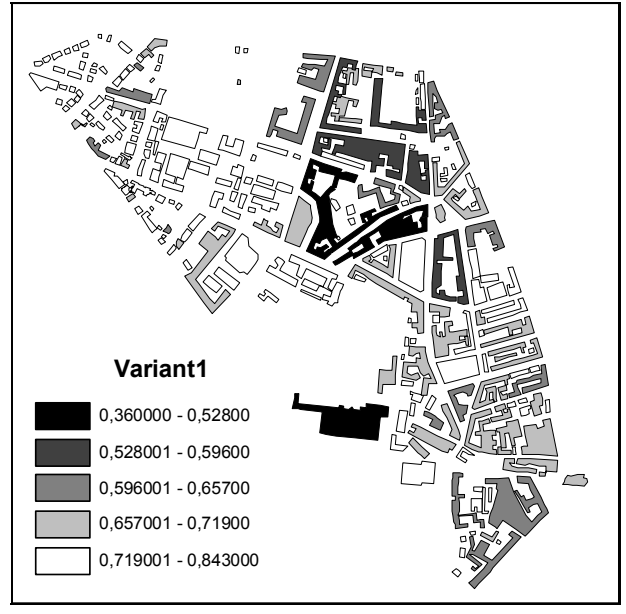

Figure 2. Quality assessment of sample (a)

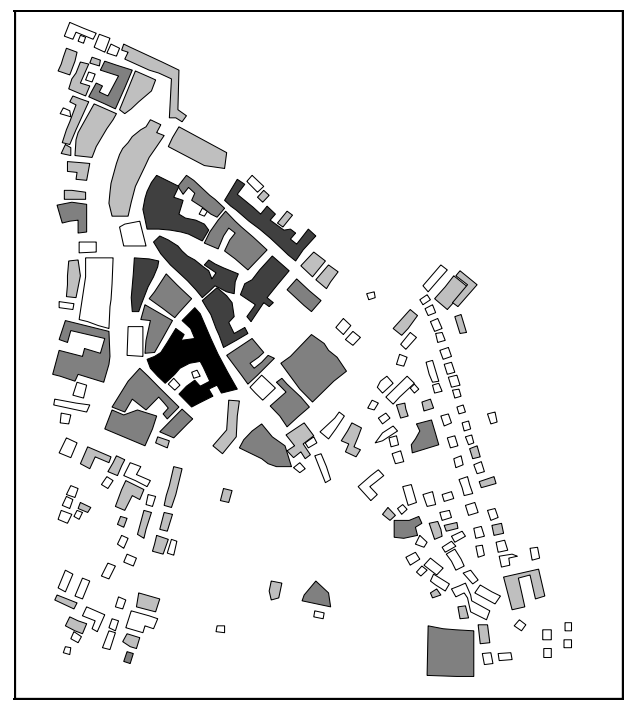

Figure 3. Quality assessment of sample (b)

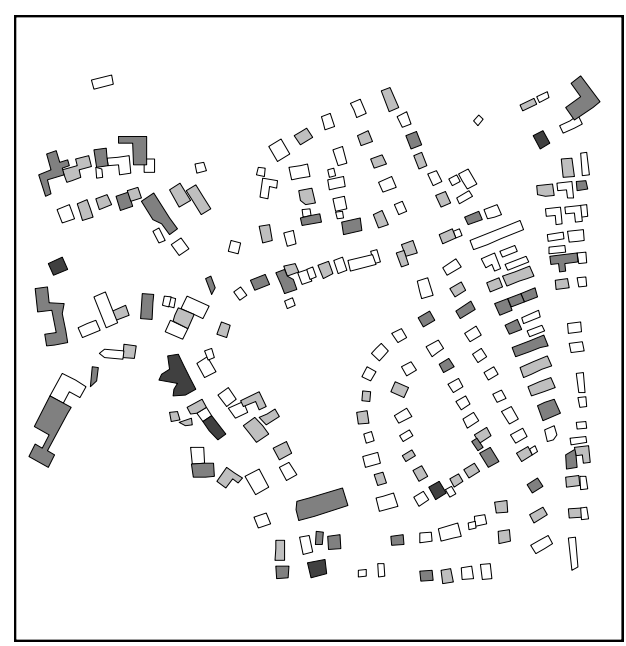

Figure 4. Quality assessment of sample (c)

Of course, visual control is one of the most important components of quality assessment. To visualize the obtained results, we display the obtained quality values with Variant 1 by different grey values for individual buildings (Figures 24). Dark grey values represent low quality, to draw the at- tention to problematic cases. Buildings with high quality measures are displayed brightly. The legend in Figure 3 applies to all three samples.

Intuitively, one would assume that the generalization of more complex buildings is more difficult. So, an automatic procedure might fail more often to produce good results. Our observation clearly confirms this presumption. The figures reveal that the quality measures depend on the complexity of the buildings: As a rule, quality scores for simple polygons are higher than for polygons with complex forms.

Table 4 illustrates the assessments for three examples that were taken from Figures 2-4. The values for the four different quality measures as well as the overall quality measure defined by the weights of Variant 1 are shown. The polygon from sample (a) has the most complex form (among the ones presented in Table 6). It was generated by aggregation of five individual buildings and subsequent simplification. The quality measures from the turning function and the reduction of vertices show that the generalization has been executed correctly.

Visually, polygons (a) and (b) have preserved their characteristic shape in the scale 1:20.000. This is reflected by a relatively high quality measure. Polygon (c) was altered rather drastically, resulting in a lower quality measure.

We have assessed the results of the obtained quality measures similarly for multiple examples by visual inspection. In our opinion, the results of Variant 1 reflect the quality of the map best. However, more tests need to be done to come to an assured conclusion about the appropriate setting of the weights.

Table 4. Quality assessment of buildings using Variant 1

\begin{tabular}{|c|c|c|c|}
\hline & $1: 10.000$ & $1: 20.000$ & $\begin{array}{c}\text { Value } \\
\text { of quality }\end{array}$ \\
\hline \multirow{6}{*}{ 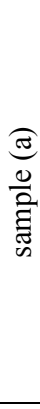 } & \multirow{6}{*}{$\begin{array}{l}N_{1}=142 \\
\text { Area }=17306.93 \\
\text { Perim }=1996.1\end{array}$} & \multirow{6}{*}{$\begin{array}{l}\mathrm{N}_{2}=84 \\
\text { Area }=13426.43 \\
\text { Perimeter }=1740.3\end{array}$} & $V_{N}=0.408$ \\
\hline & & & $V_{A}=0.952$ \\
\hline & & & $V_{P}=0.872$ \\
\hline & & & $V_{T F}=0.891$ \\
\hline & & & \\
\hline & & & $V=0.657$ \\
\hline \multirow{5}{*}{ 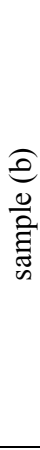 } & \multirow[b]{5}{*}{$\begin{array}{l}N_{1}=65 \\
\text { Area }=5577.31 \\
\text { Perimeter }=664.1\end{array}$} & \multirow[b]{5}{*}{$\begin{array}{l}\mathrm{N}_{2}=36 \\
\text { Area }=5570.04 \\
\text { Perimeter }=537.6\end{array}$} & $V_{N}=0.446$ \\
\hline & & & $V_{A}=0.999$ \\
\hline & & & $V_{P}=0.809$ \\
\hline & & & $V_{T F}=0.819$ \\
\hline & & & $V=0.661$ \\
\hline $\mathscr{m}$ & & & $V_{N}=0.316$ \\
\hline
\end{tabular}


The International Archives of the Photogrammetry, Remote Sensing and Spatial Information Sciences, Vol. 34, Part XXX

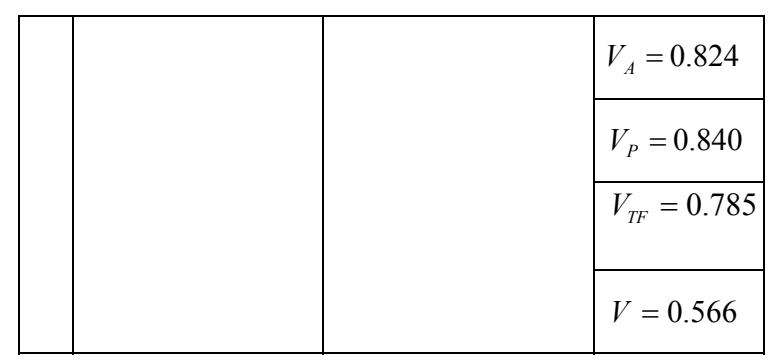

5.2 Land cover polygons from ATKIS: Aggregated DLM50 and DLM250

Land cover polygons are different from buildings in several aspects. At first, buildings are more regular, i.e., they have parallel edges and rectangular angles. Secondly, vegetation and land use polygons often form a tessellation of the plane, i.e., gaps and overlaps are not allowed. Because of this, changing the shape of a polygon is not possible without changing the neighbors shape. Due to these differences to buildings, it is necessary to conduct the quality assessment separately for both types of polygons.

Figure 5 shows polygons of the DLM50 after application of an aggregation method based on global optimization techniques (Haunert \& Wolff, 2006). The optimization criteria were compactness and semantic similarity of feature classes. We refer to this data set as "aggregated DLM50".

In order to create an appropriate representation for the target scale 1:250.000, a line simplification algorithm was applied after this aggregation, leading to the result in Figure 6. The implemented algorithm minimizes the number of polygon vertices, does not produce topological errors, and assures the new line to be within a user defined distance of the original line (de Berg et al., 1995).

The line simplification results in simple 'one-to-one' relations between features of the input data set (aggregated DLM50) and the output data set (DLM250). Calculations for the assessment of this routine were executed similarly to buildings. Results are shown in Table 5.

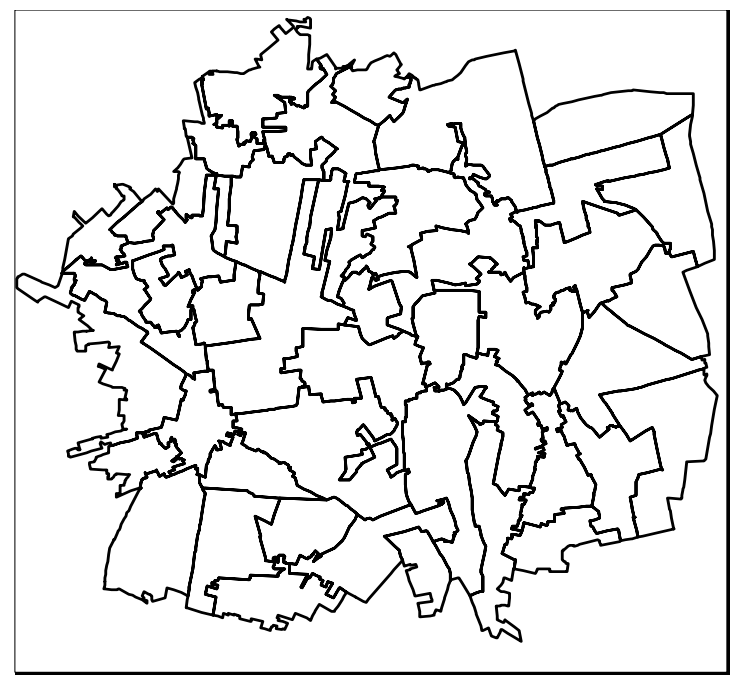

Figure 5. Source dataset DLM50

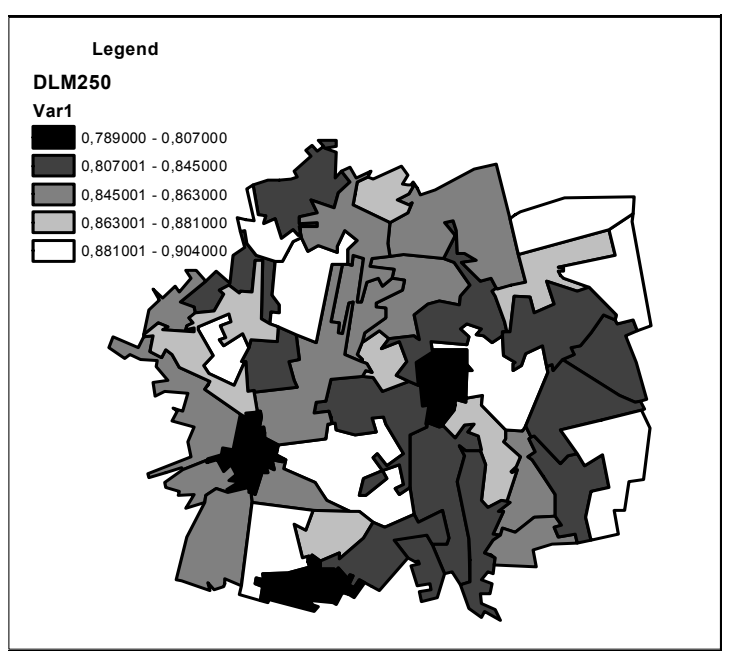

Figure 6. Quality assessment of land cover polygons (DLM250)

Table 5. Test results: Minimum, maximum and average quality for land cover polygons

\begin{tabular}{|c|c|c|}
\hline \multirow{2}{*}{ Variant 1 } & $V_{\min }$ & $V_{\max }$ \\
\cline { 2 - 3 } & 0.789 & 0.904 \\
\hline \multirow{2}{*}{$V_{a}$} & \multicolumn{2}{|c|}{$\Delta=0.115$} \\
\hline Variant 2 & 0.857 \\
\cline { 2 - 3 } & \multicolumn{2}{|c|}{$\Delta=0.107$} \\
\hline$V_{a}$ & \multicolumn{2}{|c|}{0.905} \\
\hline Variant 3 & 0.815 & 0.940 \\
\cline { 2 - 3 } & \multicolumn{2}{|c|}{$\Delta=0.125$} \\
\hline$V_{a}$ & \multicolumn{2}{|c|}{0.879} \\
\hline Variant 4 & 0.675 & 0.936 \\
\cline { 2 - 3 } & \multicolumn{2}{|c|}{$\Delta=0.261$} \\
\hline$V_{a}$ & \multicolumn{2}{|c|}{0.822} \\
\hline
\end{tabular}

Table 6. Quality assessment of land cover polygons using Variant 1

\begin{tabular}{|c|c|c|}
\hline DLM50 & DLM250 & $\begin{array}{c}\text { Value } \\
\text { of quality }\end{array}$ \\
\hline \multirow{5}{*}{$\begin{array}{l}\mathrm{N}_{1}=430 \\
\text { Area }=1415667,42 \\
\text { Perimeter }=13909\end{array}$} & \multirow{5}{*}{$\begin{array}{l}N_{2}=60 \\
\text { Area }= \\
1436249,21 \\
\text { Perimeter }=12566\end{array}$} & $V_{N}=0.833$ \\
\hline & & $V_{A}=0.994$ \\
\hline & & $V_{P}=0.972$ \\
\hline & & $V_{T F}=0.798$ \\
\hline & & $V=0.894$ \\
\hline \multirow{4}{*}{$\begin{array}{l}\mathrm{N}_{1}=96 \\
\text { Area }=1321624,61 \\
\text { Perimeter }=\end{array}$} & \multirow{4}{*}{$\begin{array}{l}\mathrm{N} 2=16 \\
\text { Area }= \\
1328953,85\end{array}$} & $V_{N}=0.860$ \\
\hline & & $V_{A}=0.986$ \\
\hline & & $V_{P}=0.903$ \\
\hline & & $V_{T F}=0.895$ \\
\hline
\end{tabular}




\begin{tabular}{|l|l|l|}
\hline 6686.67 & Perimeter $=6500,8$ & $V=0.661$ \\
\hline
\end{tabular}

Similar to the buildings is Section 5.1, the quality of individual polygons is visualized using different grey values (Figure 6). Again, the results for two examples are displayed in detail (Table 6).

We summarize these results as follows: In comparison to buildings, the average quality values are significantly higher, the variation is smaller. It is important to note, that we can not conclude from this, that the applied generalization procedure for land cover polygons is better than the method for building simplification. As mentioned earlier, important differences for both problems exist. Thus, in order to classify the results into categories such as "good" or "bad", different classification schemes need to be applied. So far, the calculated measures only allow for relative comparisons within one category of polygons.

\section{CONCLUSIONS}

A method of quality assessment for polygon generalization has been suggested and explored.

The proposed procedure offers a possibility to calculate measures for quality assessment and to visually inspect them. This allows selecting different weights for the parameters in order to highlight different preferences. The ideal is a situation, when the accumulated quality measure exactly fits the expectation of a human cartographer. This parameter setting then, in turn, can be used to quickly inspect new data sets. Although there is a correlation between the visual quality assessment and the quality value calculated with our measures, there is still room for improvement. Obviously, the measures fit more to the manmade objects and less to the natural ones.

We have considered geometrical quality measures of polygon generalization from the point of view the reducing the amount of data and keeping the map similar to the input map. Results of quality assessment for the buildings (cadastral data) and land cover polygons (ATKIS data) are received.

Future research on map quality will be along the four directions:

- developing methods for quality assessment of generalization $n$-polygons into $m$-polygons.

- developing measures on meso and macro level;

- developing quality measures for interrelations of one object type (for example, buildings) with other types (roads and rivers);

- developing complex quality assessment methods for a group of objects with combination of metric, semantic and topological information;

\section{REFERENCES}

Anders, K.-H, 2004. Parameterfreies hierarchisches GraphClustering-Verfahren zur Interpretation raumbezogener Daten. Dissertation, Universität Stuttgart. Persistent Identifier: urn:nbn:de:bsz:93-opus-20249

Anders, K.-H., 2006. Grid Typification. In: Riedl, A., Kainz, W. \& Elmes, G.A. (ed.) Progress in Spatial Data Handling, 12th International Symposium on Spatial Data Handling. Springer-Verlag, 2006, pp. 633 - 642.

Bard, S., 2004. Quality assessment of cartographic generalization. Transactions in GIS, 8(1), pp. $63-81$.

Bard, S., Ruas A., 2004. Why and how evaluating generalized data? In: Proceedings of 12th International Symposium. Progress in Spatial Data Handling. Springer. pp. 327 - 342.

Burrough, P., McDonnell, R., 1998. Principles of geographical Information systems. Oxford University Press. p. 223.

de Berg, M., van Kreveld, M., Schirra., S., 1995. A new approach to subdivision simplification. In: Twelfth International Symposium on Computer-Assisted Cartography, volume 4, pp. 79-88, Charlotte, North Carolina, USA.

Cheng, T., Li, Zh., 2006. Toward quantitative measures for the semantic quality of polygon generalization. Cartographica, 41(2), pp. $135-147$.

Frank, R., Ester, M., 2006. A quantitative similarity measure for maps. In: Proceedings of 12th International Symposium. Progress in Spatial Data Handling. Springer. pp. 435 - 450.

Galanda, M., 2003. Automated polygon generalization in a multi agent system, PhD Thesis, Department of Geography, University of Zurich, Switzerland.

Garaevskaya, L. S., Malusova, N. V., 1990. The practical manual on cartography. Nedra, Moscow, pp. 46-47.

Guptill, C., Morrison J. L., 1995. Elements of spatial data quality. Elsevier Science Ltd. p. 202.

Haunert, J.-H., Wolff, A., 2006. Generalization of land cover maps by mixed integer programming. In: Proceedings of 14th International Symposium on Advances in Geographic Information Systems, 10. - 11. November 2006, Arlington, Virginia, USA. pp. $75-82$.

Heinzle, F., Anders, K.-H. \& Sester, M., 2006. Pattern Recognition in Road Networks on the Example of Circular Road Detection. In: Raubal, M., H.J. Miller, A.U. Frank, M.F. Goodchild (Eds.): Geographic Information Science, GIScience 2006, Münster, Germany. LNCS 4197, pp. 253267.

Joao E., M., 1998: Causes and Consequences of Map Generalization, Taylor \& Francis, p.220.

Kolokolova, I., V. 2005. Automatic and interactive quality assessment in technologies of automated generalization. In: Proceedings of scientific congress "GEO-Sibir 2005". 25.-29. April 2005, Novosibirsk, Russia. pp. 287-290.

Mayberry, M., (08 Sep. 2002). Paper "Data quality: Before the map is produced" http://www.directionsmag.com/article.php?article_id $=250$

Müller, J. C., Weibel, R., Lagrange J. P., Salge F., 1995. Generalization: state of the art and issues. GIS and generalization. Methodology and practice. GIS Data I. Taylor \& Francis. pp. $3-17$ 
Peter, B., 2001. Measures for the generalization of polygonal maps with categorical data. Fourth ICA Workshop on progress in Automated Map Generalization, 2.-4. August 2001, Beijing, China.

Peura, M., Iivarinen, J., 1997. Efficiency of simple shape descriptors. In 3rd International Workshop on Visual Form, Capri, Italy.

Rodriguez, A. and Egenhofer, M., 2004. Comparing geospatial entity classes: an asymmetric and context dependent similarity measure. Geographical Information Science, 18(3):229-256.

Skopeliti, A., Tsoulos, L., 2001. The accuracy aspect of cartographic generalization. In: Proceedings of the GIS Research UK 9th Annual Conference GISRUK 2001. Wales, UK, pp. 22-25.

van Smaalen, J. W. N., 2003. Automated aggregation of geographic objects. A new approach to the conceptual generalization of geographic databases. Doctoral Dissertation. Wageningen University. The Netherlands.

TSNIIGAiK, 2003. OST 68-3.4.2-2003 The standard of branch. Digital maps. Methods of estimation of data quality. The general requirements. Moscow, Russia.

http://gis-lab.info/docs.html

Zahn, C. T., Roskies, R. Z., 1977. Fourier descriptors for plane closed curves. IEEE Transactions on Computers, C21(3):269-281.

\section{ACKNOWLEDGEMENTS}

All colleagues at the Institute of Cartography and Geoinformatics, Leibniz Universität Hannover for providing a friendly and positive working atmosphere with useful discussions are thanked.

The funding of Mikhail Lomonosov program from German Academic Exchange Service (DAAD) and Russian Ministry of Education and Science is gratefully acknowledged. 